\title{
Characteristics of boundary layer flow induced by a solitary wave
}

\author{
C. Lin ${ }^{1}$, S. C. Hsieh ${ }^{1}$, S. M. Yu ${ }^{1} \&$ R. V. Raikar ${ }^{2}$ \\ ${ }^{I}$ Department of Civil Engineering, National Chung Hsing University, \\ Taiwan \\ ${ }^{2}$ Department of Civil Engineering, \\ K. L. E. S. College of Engineering and Technology, India
}

\begin{abstract}
The experimental results on the characteristics of bottom boundary layer flow induced by a solitary wave, obtained using high speed particle image velocimetry (HSPIV) and particle trajectory techniques, are presented in the paper. The particle trajectory technique is particularly used near the bottom boundary to visualize the flow field. The velocity distribution in the bottom boundary layer is classified into two types from the point of view of similarity analysis: one pre-passing and another post-passing of the crest of solitary wave across the measuring section. The velocity distribution in the bottom boundary layer before the passing of solitary wave-crest was regressed using two hyperbolic tangent functions. The free stream velocity $u_{\infty}$ and the boundary layer thickness $\delta$ are found to be the suitable characteristic velocity and length scales to obtain the similarity profile. However, other characteristic properties of the boundary layer such as displacement thickness, momentum thickness and energy thickness were also used in the analysis, but they are proved to be mere multiples of boundary layer thickness $\delta$. On the other hand, to attain the similarity profile for the velocity distribution for post-passing of the crest of solitary wave, the characteristic velocity scale $\left(u_{\infty}-U_{m}\right)$, where $U_{m}$ is the maximum negative velocity, and length scale: maximum thickness of reverse flow $b_{0}$ is used.
\end{abstract}

Keywords: bottom boundary flow, solitary waves, flow visualization, high speed particle image velocimeter, similarity profile. 


\section{Introduction}

Solitary waves as first observed by Russell [1] can travel over large distances and exhibit the property of stable state of motion. They propagate steadily without either steepening their wave height or widening their wave length but maintaining constant wave length and relative wave height. In addition, they also do not loose their energy so much and structure as well. These solitary waves to some extent resemble to a tsunami because of shallow water depth, before they undergo changes near the shores. Therefore, the solitary waves are often studied due to their simple and permanent wave form and also the study of solitary waves (or combinations of negative and positive solitary-like waves) help in simulating the run-up and shoreward inundation. Keulegan [2] was the pioneer to present the effects of viscous damping on solitary waves. Later, Mei [3] used perturbation method to obtain the similar results. However, the study of Keulegan [2] and Mei [3] mainly concentrate on the estimation of damping rate. Recently, Liu and Orfila [4] obtained analytical solutions for viscous boundary layer flows under transient long waves, including the damping rates for the solitary waves. Further, the perturbation analysis of Liu and Orfila [4] for linear boundary layer solution was extended by Liu et al. [5] for fully nonlinear boundary layer condition.

The objective of the present study is to emphasize mainly on the measurement of flow structure within the bottom boundary layer induced by a solitary wave using HSPIV and particle trajectory techniques. The particle trajectory technique is particularly used to enhance the flow field near the bottom boundary layer.

\section{Experimentation}

\subsection{Experimental setup and conditions}

A glass-walled and glass-bottomed wave flume $14 \mathrm{~m}$ long, $0.25 \mathrm{~m}$ wide and 0.5 $\mathrm{m}$ deep located at the Hydraulics Laboratory of the Department of Civil Engineering, National Chung Hsing University, Taiwan was used to conduct the experiments. The wave flume was fitted with a piston-type wave maker at one of its end, which was driven by a servo motor; while at the other end the wave dissipater was fixed to dampen the waves. An electromagnetic clutch installed inside the wave maker allowed a rapid start and stop of the wave paddle thereby facilitating the generation of the desired solitary waves. The servo motor used to trigger the wave paddle made it possible to generate an individual solitary wave without followed by any dispersive tail wave train. The validation of generated solitary waves is discussed later. However, the generated solitary waves, being highly repeatable, were clean enough with certain combinations of wave height and water depth.

The measuring section was located at $8 \mathrm{~m}$ from the wave board and two fields of view (FOV) were used for the flow visualization. FOV A located near the boundary area has the size of $2 \mathrm{~cm} \times 1 \mathrm{~cm}$ with $1 \mathrm{~cm}$ of its width on either side of the measuring section and FOV B with its effective size $12 \mathrm{~cm} \times 12 \mathrm{~cm}$ 
covering the potential area. Fig. 1 shows the schematic view of the wave flume with location of measuring section, two FOV's and the coordinate system used in the study. The origin of the coordinate system is located at the bottom center of the FOV A with $X$ measured along the flume bed in the direction of the wave motion and $Y$ normal to the flume bed. The time $t=0$ indicated the moment when the crest of the solitary wave reaches the position $X=0$. In all 7 experimental runs were taken with the wave height $H$ in the range between 1.1 $\mathrm{cm}$ to $3 \mathrm{~cm}$, water depth $h$ between $7 \mathrm{~cm}$ to $10 \mathrm{~cm}$ so that the relative wave height ratio $H / h$ is between 0.11 to 0.414 . Table 1 furnishes the details of all experimental runs. In the present study, the maximum Reynolds number defined by $\left(R_{e}\right)_{\max }=U h / v$ is about 18,750 , where $U$ is the maximum free stream velocity at the edge of bottom boundary layer [i.e., $\left(u_{\infty}\right)_{\max }$ ], and $v$ is the kinematic viscosity.

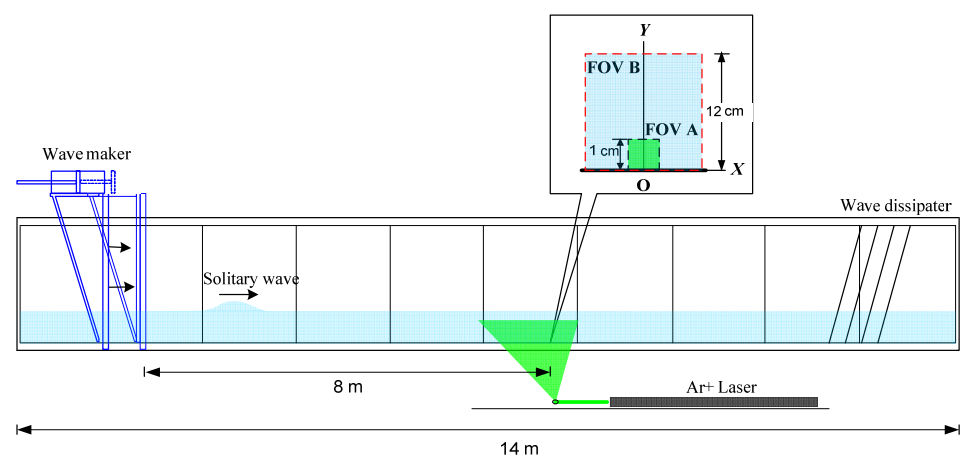

Figure 1: Schematic view of wave flume showing location of measuring section, FOV's and coordinate system.

Table 1: $\quad$ Details of experimental conditions.

\begin{tabular}{|c|c|c|c|c|}
\hline Case & $\begin{array}{c}\text { Wave height } \\
H \\
(\mathrm{~cm})\end{array}$ & $\begin{array}{c}\text { Water depth } \\
h \\
(\mathrm{~cm})\end{array}$ & $\frac{H}{h}$ & $\begin{array}{c}\text { Measured wave celerity } \\
c \\
(\mathrm{~cm} / \mathrm{s})\end{array}$ \\
\hline $\mathrm{A}$ & 1.1 & 10 & 0.110 & 108.42 \\
\hline B & 1.1 & 7 & 0.157 & 92.79 \\
\hline $\mathrm{C}$ & 1.4 & 7 & 0.200 & 93.44 \\
\hline $\mathrm{D}$ & 2.0 & 8 & 0.250 & 100.44 \\
\hline $\mathrm{E}$ & 1.9 & 7 & 0.271 & 92.48 \\
\hline $\mathrm{F}$ & 3.0 & 8 & 0.375 & 102.28 \\
\hline $\mathrm{G}$ & 2.9 & 7 & 0.414 & 96.14 \\
\hline
\end{tabular}




\subsection{Velocity measurement}

HSPIV system used to measure the velocity field. The laser light sheet spread for the flow visualization is made use of in HSPIV. The image recording system was a 10 bit Complementary Metal-Oxide (CMO) high speed camera (Phantom V5.1) having resolution of $1024 \times 1024$ pixel and $1200 \mathrm{~Hz}$ maximum framing rate (aperture opening rate of $10-47000 \mu \mathrm{s}$ ). Velocity fields were determined by cross-correlation analysis using commercial software (TSI Insight). The interrogation windows of $32 \times 32$ pixels with $50 \%$ overlap were used in the cross-correlation analysis. In parallel to HSPIV system, a fiber laser Doppler velocimeter (FLDV) was also used to verify the velocity field measured by HSPIV system.

\subsection{Validation of solitary wave generation}

The wavemaker was fitted with high precision servo motor system, which could generate a perfect solitary wave nearly without any dispersive tail wave train. The generated solitary waves in this study followed the procedure described by Goring [6]. Initially, the validation of the solitary wave generation by the wavemaker system was carried out for the experimental case D having wave height $H=2.0 \mathrm{~cm}$ and water depth $h=8 \mathrm{~cm}$. Along with the comparison of free surface elevation, the associated wave celerity was also assessed. Fig. 2(a) presents the comparison of free surface profile between the generated solitary wave and theoretical wave form of

$$
\eta(X, t)=H \sec h^{2}\left[\sqrt{\frac{3 H}{4 h^{3}}}(X-c t)\right]
$$
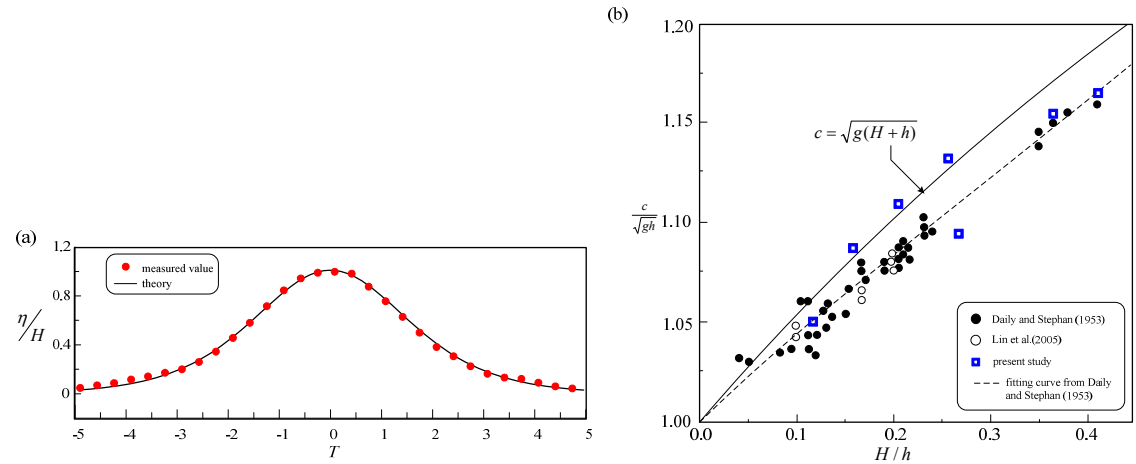

Figure 2: Validation of target solitary wave for experimental case D: (a) comparison of free surface profile; (b) comparison of wave celerity. 
where $c=$ wave celerity, can be obtained as $\sqrt{g(H+h)}$. It is interesting to observe a good conformity of the generated wave with the theoretical one. Fig. 2(b) shows the comparison of the wave celerity. There is a satisfactory agreement between the generated waves and the data of Daily and Stephan [7] and Lin et al. [8], both exhibiting slightly lower celerity than the theoretical.

\section{Result and discussions}

The images obtained by particle trajectory technique using the high speed digital camera in the boundary area of size $2 \mathrm{~cm} \times 1 \mathrm{~cm}$ (FOV A) with $1 \mathrm{~cm}$ width on either side of the measuring section for the non-dimensional time $-3.0 \leq T \leq 4.0$ are captured. The bottom boundary layer can be distinctly visualized in these images. It is supported by the corresponding phase-averaged velocity field depicted in Fig. 3 for $T=-3.0 \sim 4.0$. The velocity field looks similar to the free stream velocity up to $T<1.00$; however, for $T \geq 2.00$ it is interesting to observe the reversal of flow velocity. The velocity distribution after $T \geq 2.00$ resembles that of velocity profile due to wall jet. This indicates the development of negative or adverse pressure gradient after passing of the solitary wave at the measuring section $(T=0)$, particularly after $T \geq 1.00$. The exact non-dimensional time $T$, when the flow reversal occurs and adverse pressure gradient develops at the measuring section can be viewed by Fig. 4, which illustrates the velocity field in potential area at the measuring section for $-5.0 \leq T \leq 5.0$ for experimental case D. From Fig. 4, the occurrence of flow reversal can be found to be at $T=$ 1.60 at location very close to the boundary. However, the zone of flow reversal increases with the passage of solitary wave (for $T \geq 1.60$ ) as seen from Fig. 4. The vertical position of center of flow reversal near the boundary is found to be within $Y / h<0.025$. Fig. 4 also depicts another horizontal scale $5.0 \geq X / h \geq-5.0$ corresponding to the same velocity field. However, the values of $X / h$ are obtained by parametric transformation using the theoretical wave form (Eq. (1)). Using this $X / h$ scale, it could be seen that the position where the flow reversal occurs is at $X / h=-2.00$.
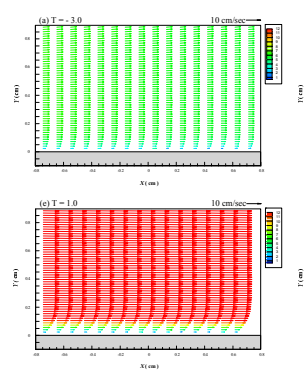
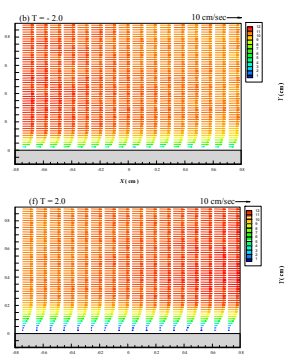
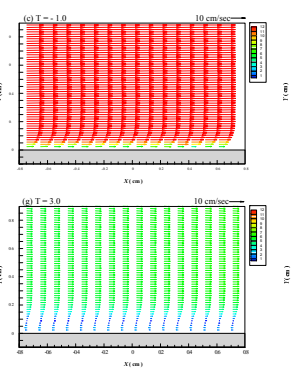
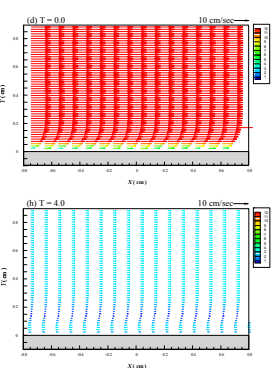

Figure 3: HSPIV velocity vectors measured near and in the boundary area for case $\mathrm{D}$ at non-dimensional time $T$ : (a) -3.0 ; (b) -2.0 ; (c) -1.0 ; (d) 0.0 ; (e) 1.0 ; (f) 2.0; (g) 3.0; (h) 4.0. 


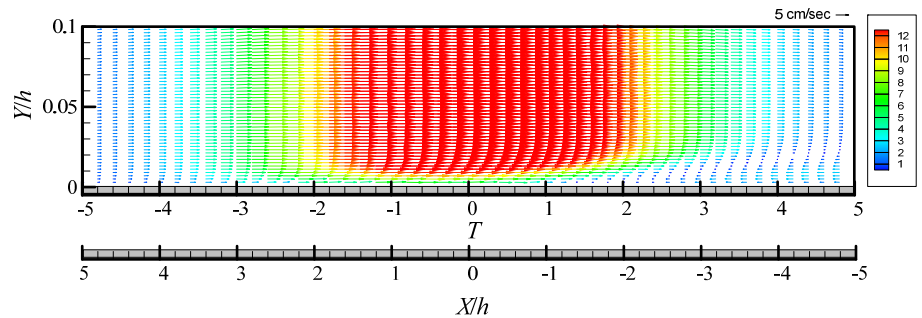

Figure 4: Velocity field obtained at measuring section near and in the boundary area for case $\mathrm{D}$ at $-5.0 \leq T \leq 5.0$.

By obtaining the non-dimensional time $T$ and position $X / h$ of occurrence of adverse pressure gradient (flow reversal) for different experimental cases, their variation is plotted as shown in Fig. 5. Fig. 5(a) presents the variation of nondimensional time $T$ of occurrence of adverse pressure gradient with the relative height $H / h$. It shows a linear relationship between non-dimensional time $T$ and relative height $H / h$, depicting the decrease in $T$ with increase in relative height $H / h$ following Eq. (2). On the other hand, the variation between the position $X / h$ of occurrence of adverse pressure gradient with relative height $H / h$ is plotted in Fig. 5(b). Fig. 5(b) indicates the increase in $X / h$ with increase in relative height $H / h$ following Eq. (3).

$$
\begin{aligned}
& T=-1.794\left(\frac{H}{h}\right)+2.315 \\
& \frac{X}{h}=2.366\left(\frac{H}{h}\right)-2.717
\end{aligned}
$$

The distribution of horizontal velocity $u$ measured by HSPIV at the measuring section for the experimental case $\mathrm{D}$ with non-dimensional $-5.0 \leq T \leq$ 5.0 at every $T=0.5$ are presented in Fig. 6 . The movement of solitary wave at the measuring section is illustrated in Fig. 6(a) showing various $T(=0.5)$ at points numbered 1 to 21 . The horizontal velocity profiles for pre-passing of the solitary wave $(-5.0 \leq T \leq 0.0)$ at the measuring section are shown in Fig. 6(b) as indicated by number $1-11$ and for post-passing of the solitary wave $(0.0 \leq T \leq$ 5.0) in Fig. 6(c) with number $11-21$. It is interesting to observe that the horizontal velocity profiles follow certain specific trends for each of the case of pre-passing and post-passing of solitary wave. Hence, they are grouped into two classes, which would help in attaining the similarity profiles.

From horizontal velocity profiles shown in Fig. 6(b) before passing of the solitary wave $(T \leq 0.0)$ at the measuring section, it can be seen that they follow a particular trend of increasing horizontal velocity $u$ and then reaching a uniform distribution representing the free stream velocity after certain value of $Y$. The 
position $Y$ corresponding to the location of free stream velocity $u=u_{\infty}$ depends on $T$. However, the distance $Y$ increases with increase in $T$ approximately from $Y$ $=0.1 \mathrm{~cm}$ (at $T=-5.00)$ to $0.25 \mathrm{~cm}($ at $T=0)$. The corresponding value of free stream velocity are $u_{\infty}=1.2 \mathrm{~cm} / \mathrm{s}$ at $T=-5.00$ to $u_{\infty}=20 \mathrm{~cm} / \mathrm{s}$ at $T=0$. That is the free stream velocity $u_{\infty}$ increases with the approach of solitary wave towards the test section and reaches its maximum value $\left(u_{\infty}\right)_{\max }$. This can be evidenced from Fig. 6(b) in which the free stream velocity at non-dimensional time $T, u_{\infty}(T)$ increases as $T$ increases from -5.0 to 0.0 .

The profiles of horizontal velocity $u$ after passing of the solitary wave $(T \geq$ $0.0)$ are shown in Fig. 6(c). After passing of the solitary wave $(T \geq 0.0)$, the profiles of horizontal velocity $u$ continue to exhibit the same trend as those of pre-passing of solitary wave $(T \leq 0.0)$. However, the free stream velocity $u_{\infty}$ starts decreasing with increase in $T(0.0 \sim 2.0)$. After $T \geq 2.0$, the profiles of horizontal velocity $u$ depict the flow reversal within the zone close to the boundary. In this zone, the horizontal velocity $u$ increases in the negative direction reaching its maximum negative value $U_{m}$ and then decreases to become positive and increases towards the top to reach free stream velocity $u_{\infty}$. It is pertinent to mention that the free stream velocity at non-dimensional time $T$, $u_{\infty}(T)$ continues to decrease with $T$ and reaches its minimum value $\left(u_{\infty}\right)_{\text {min }}$. The characteristic nature of the velocity distribution is similar to that of the case of wall jet. The thickness of this zone where the velocity reversal occurs, the location of maximum negative value $U_{m}$ from the boundary and the magnitude of $U_{m}$ depend on the non-dimensional time $T$.
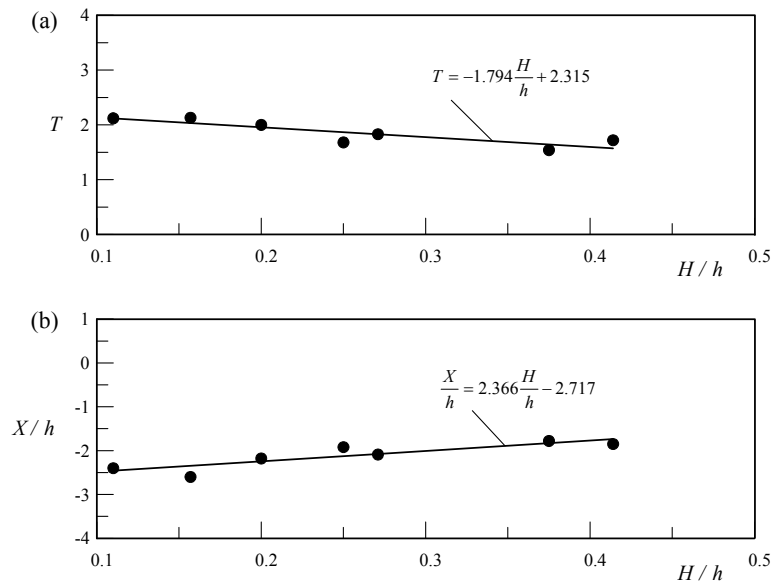

Figure 5: (a) Dependency of non-dimensional time $T$ of occurrence of adverse pressure gradient on the relative height $H / h$; (b) dependency of non-dimensional position $X / h$ of adverse pressure gradient on the relative height $H / h$. 
The nonlinear regression analysis was performed for the measured velocity profiles to obtain the equation for the horizontal velocity $u$ distributions. The distribution of the horizontal velocity $u$ in the boundary layer was classified into two types as mentioned in the previous section: one for the pre-passing of the solitary wave $(T \leq 0.0)$ and another for post-passing of the solitary wave $(T \geq$ $0.0)$ at the section.

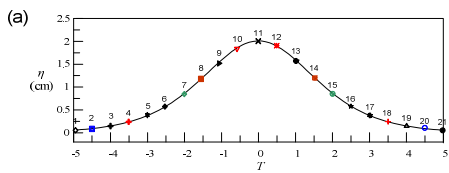

(b)

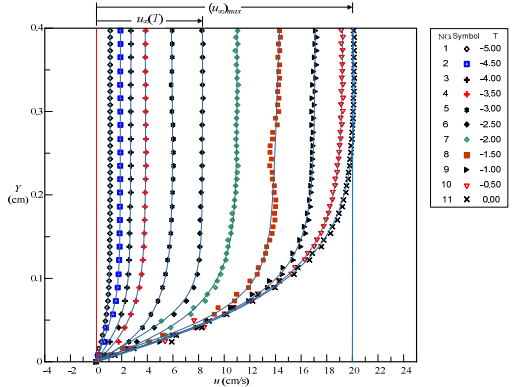

(c)

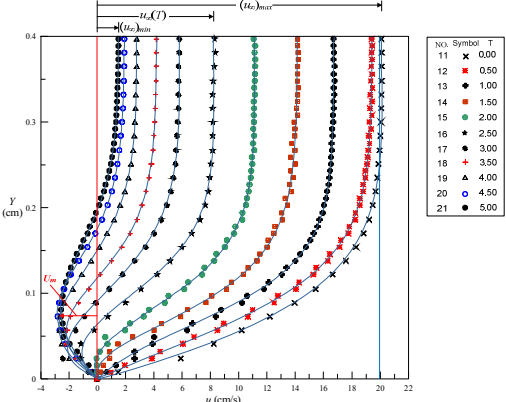

Figure 6: (a) Solitary wave showing the measuring instants of horizontal velocity; (b) horizontal velocity $u$ profiles at measuring section for pre-passing of solitary wave $(T \leq 0.0)$; (c) horizontal velocity $u$ profiles at measuring section for post-passing of solitary wave $(T \geq$ 0.0 ), for case D.

By observing the velocity profiles shown in Fig. 6(b), the characteristic nature of velocity profile is sketched as illustrated in Fig. 7(a). The horizontal velocity $u$ increases in the boundary layer and reaches free stream velocity $u_{\infty}$ at the end of boundary layer. To obtain the velocity distribution for the horizontal velocity $u$ for the pre-passing of the solitary wave $(T \leq 0.0)$, three types of nonlinear regression equations having two hyperbolic tangent functions (Eq. (4)) were attempted.

$$
\begin{gathered}
u(Y)=C_{1} \times\left[\tanh \left(C_{2} Y+C_{3}\right)-\tanh \left(C_{3}\right)\right] \\
u(Y)=C_{4} \times \tanh \left(C_{5} Y^{2}+C_{6}\right)-\tanh \left(C_{7} Y^{2}+C_{8}\right) \\
u(Y)=C_{9} \times \tanh \left(C_{10} Y+C_{11}\right) \times \tanh \left(C_{12} Y+C_{13}\right)+C_{14}
\end{gathered}
$$

where $C_{1}$ to $C_{14}$ are constants. Amongst these three equations, Eq. (4c) is found to be the most appropriate one to fit all the measured velocity profiles before 
passing of the solitary wave $(T \leq 0.0)$. Hence, Eq. (4c) is considered as the suitable velocity distribution for the horizontal velocity $u$ for pre-passing of the solitary wave having $\mathrm{R}^{2}$ value of fitting as 0.993 . Fig. 6 (b) also demonstrates the satisfactory fitting of the velocity profiles obtained by Eq. (4c) to the experimental data.

(a)

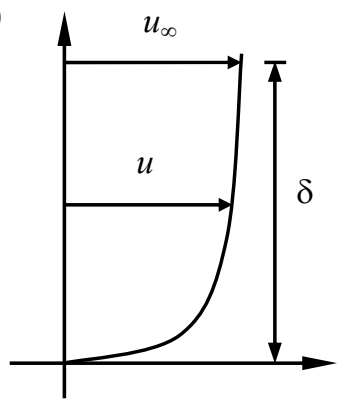

(b)

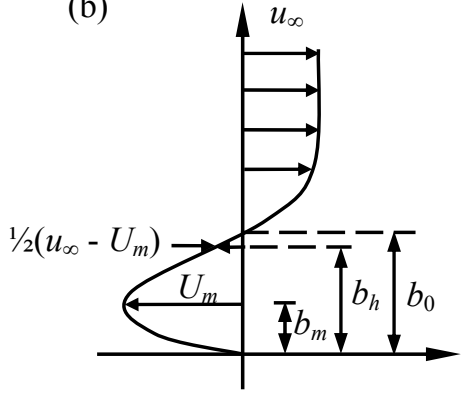

Figure 7: Schematic diagram illustrating characteristic profiles of horizontal velocity: (a) for pre-passing of solitary wave $(T \leq 0.0)$; (b) for postpassing of solitary wave $(T \geq 0.0)$.

On the other hand, for post-passing of solitary wave $(T \geq 0.0)$ at the section, the velocity profiles gradually change their trend as a result of flow reversal that occurs near the boundary for $T$ exceeding nearly 1.60 (depends on the experimental case). From Fig. 6(c) for experimental case D, it can be evidenced that the horizontal velocity profile at $T=2.00$ shows the distinct reversal of flow. After $T \geq 2.00$, all profiles follow the same trend. However, as $T$ increases the maximum negative value $U_{m}$ increases, while the free stream velocity at nondimensional time $T, u_{\infty}(T)$ decreases. The value of $U_{m}$ reaches its maximum value corresponding to non-dimensional time $T=5.00$ and correspondingly, the free stream velocity reaches its minimum value $\left(u_{\infty}\right)_{\text {min }}$. The details are illustrated in Fig. 6(c). Following this nature of velocity profile, the definition sketch illustrating the characteristic of the horizontal velocity after passing of solitary wave at the test section is drawn as shown in Fig. 7(b). In Fig. 7(b), $b_{m}$ represents the height of the maximum negative velocity, $b_{h}$ represents half-velocity-deficit [where $u=\left(u_{\infty}-U_{m}\right) / 2$ ] and $b_{0}$ represents maximum thickness of reverse flow. To match this specific nature of horizontal velocity, the nonlinear equation consisting of a hyperbolic tangent function and a cosine function of the form Eq. (5) is selected to attain the velocity distribution. It is very interesting to mention that it fits very well for the horizontal velocity $u$ profiles after passing of the solitary wave with its $\mathrm{R}^{2}$ value 0.95 .

$$
u(Y)=C_{15} \times\left[\tanh \left(C_{16} Y+C_{17}\right)-C_{15}\right] \times \cos \left(C_{18} Y+C_{19}\right)+C_{20}
$$

where $C_{15}$ to $C_{20}$ are constants. The fitting of the velocity profiles to the experimental data are represented in Fig. 6(c). By observing the similar trend of 
velocity profiles before passing of the solitary wave $(T \leq 0.0)$ at the section presented in Fig. 6(b), the fitting of the similarity profile for the horizontal velocity $u$ distribution was thought of. To decide on the length scale for obtaining the similarity profile, four length scales corresponding to boundary layer theory, such as boundary layer thickness $\delta$, displacement thickness $\delta$, momentum thickness $\theta$ and energy thickness $\delta_{e}$ were considered. On the other hand, the free stream velocity $u_{\infty}$ at the edge of bottom boundary layer is used as characteristic velocity. Fig. 8 show the similarity profile for the horizontal velocity $u$ considering boundary layer thickness $\delta$. The corresponding regression equation is expressed as in Eq. (6).

$$
\frac{u}{u_{\infty}}=1-\exp \left[-4.29\left(\frac{Y}{\delta}\right)\right]
$$

By observing the similarity profile and considering various conditions, the authors found that the boundary layer thickness $\delta$ and the free stream velocity $u_{\infty}$ are the appropriate characteristic length and velocity scales for defining the similarity profile for the horizontal velocity $u$ before passing of the solitary wave $(T \leq 0.0)$ at the section.

Similarly, to obtain the similarity profile for the horizontal velocity $u$ after passing of the solitary wave $(T \geq 0.0)$, three length scales such as height of the maximum negative velocity $b_{m}$, half-velocity-defect $b_{h}$ and maximum thickness of reverse flow $b_{0}$ were considered. Along with these, the velocity defect $\left(u_{\infty}-\right.$ $U_{m}$ ) is used as the velocity scale. However, it is important to mention that the velocity profiles those depict the flow reversal were considered in obtaining the similarity profile. Out of the three length scales attempted, the maximum thickness of reverse flow $b_{0}$ was found to give good result with the respective $\mathrm{R}^{2}$ value being 0.998 . The similarity profile is defined by Eq. (7).

$$
\begin{array}{r}
\frac{u-U_{m}}{u_{\infty}-U_{m}}=[-1.78 \times \\
\left.\times \tanh \left(1.33 \frac{Y}{b_{0}}-0.35\right)+1.78\right] \\
\times \cos \left(-1.40 \frac{Y}{b_{0}}-1.77\right)+1.00
\end{array}
$$

Fig. 9 presents the similarity profile for the horizontal velocity $u$ for postpassing of the solitary wave $(T \geq 0.0)$ with respective length scale as maximum thickness of reverse flow $b_{0}$ and $\left(u_{\infty}-U_{m}\right)$ as the velocity scale. Both the similarity profiles shown depict the promising self-preservation, except slight variation in $\mathrm{R}^{2}$ value. However, as the horizontal velocity profiles are comparable with those of the case of a wall jet, the flow phenomenon within boundary layer after passing of the solitary wave (with velocity profiles depicting the flow reversal) could be related to wall jets. With this point of view, the maximum thickness of reverse flow $b_{0}$ is considered as the most suitable 
length scale. Hence, Eq. (7) is considered as the ideal similarity profile for postpassing of solitary wave $(T \geq 0.0)$ with the velocity defect $\left(u_{\infty}-U_{m}\right)$ and the thickness of maximum reverse flow $b_{0}$ as the characteristic velocity and length scales.

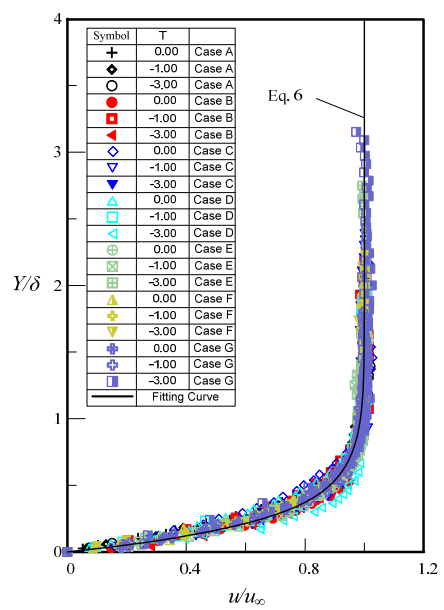

Figure 8: $\quad$ Similarity profile for horizontal velocity $\mathrm{u}$ for pre-passing of solitary wave $(T \leq 0.0)$ with the length scale using boundary layer thickness $\delta$.

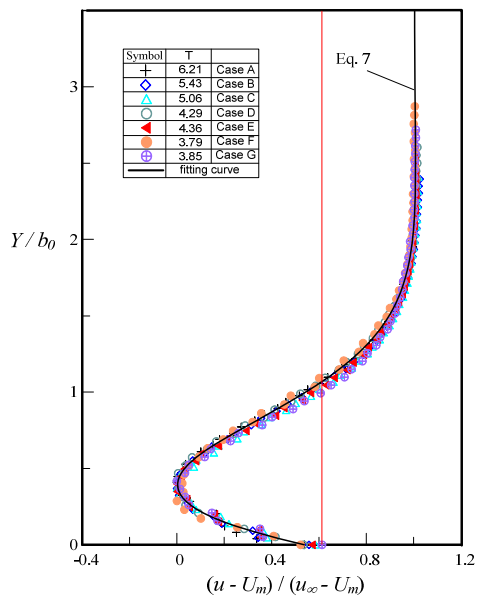

Figure 9: Similarity profile for horizontal velocity $\mathrm{u}$ for post-passing of solitary wave $(T \geq 0.0)$ with length scale using maximum thickness of reverse flow $b_{0}$. 


\section{Conclusions}

The characteristics of bottom boundary layer flow induced by a solitary wave are investigated using high speed particle image velocimetry (HSPIV) and particle trajectory techniques. The important results are summarized as follows:

1. Based on the nature of horizontal velocity profiles in the bottom boundary layer, the velocity distribution is classified into two types: one for pre-passing and another for post-passing of the crest of solitary wave across the measuring section.

2. Two hyperbolic tangent functions were used in nonlinear regression analysis to fit the anticipated velocity distribution to the measured horizontal velocity profiles in the bottom boundary layer before passing the crest of solitary wave. Further, to obtain the similarity profile for the velocity distribution, various characteristics properties of the boundary layer such as boundary layer thickness, displacement thickness, momentum thickness and energy thickness were considered as length scales in the analysis along with the free stream velocity $u_{\infty}$ as velocity scale. However, the boundary layer thickness $\delta$ and the free stream velocity $u_{\infty}$ are found to be the appropriate characteristic length and velocity scales to obtain the similarity profile. Other length scales (displacement thickness, momentum thickness and energy thickness) proved to be simple multiple of boundary layer thickness.

3. On the other hand, for post-passing of the crest of solitary wave, the velocity profile is fitted using both hyperbolic tangent and cosine functions in the nonlinear regression analysis. In addition, the height of maximum negative velocity $b_{m}$, maximum thickness of reverse flow $b_{0}$ and half-velocity-defect $b_{h}$ were considered as length scales along with velocity deficit $\left(u_{\infty}-U_{m}\right)$ as the velocity scale, in attaining the similarity profile. Amongst the three length scales, the maximum thickness reverse flow $b_{0}$ and half-velocity-deficit $b_{h}$ are found to be suitable. Considering the flow phenomena after passing of the solitary wave following the trend of wall jets, the maximum reverse flow layer thickness $b_{0}$ is considered as the appropriate length scale. Hence, the similarity profile is obtained using $b_{0}$ and $\left(u_{\infty}-U_{m}\right)$ as the characteristic length and velocity scales.

\section{Acknowledgement}

The writers gratefully acknowledge the support of this work by National Science Council, Taiwan, under Grant Nos. NSC 96-2221-E-005-078-MY3 and NSC 992811-E-005-003.

\section{References}

[1] Russell, J. S. "Report of the committee on waves", Rep. $7^{\text {th }}$ Meet. Brit. Assoc. Adv. Sci., Liverpool, John Murray, London, 417-496, 1838. 
[2] Keulegan, G. H., Gradual damping of solitary wave. Journal of Research, National Bureau of Standards, 40, pp. 607-614, 1948.

[3] Mei, C. C., The applied dynamics of ocean surface waves. John Wiley and Sons, 1983.

[4] Liu, P. L. F., and Orfila, A., Viscous effects on transient long waves. Journal of Fluid Mechanics, 520, pp. 83-92, 2004.

[5] Liu, P. L. -F., Park, Y. S., and Cowen, E. A., Boundary layer flow and bed shear stress under a solitary wave. Journal of Fluid Mechanics, 574, pp.449463, 2007.

[6] Goring, D. G., Tsunamis - The propagation of long waves onto a shelf. Rep. No. KH-R-38, California Institute of Technology, Pasadena, California, 1978.

[7] Daily, J. W., \& Stephan, S. C., Characteristics of solitary wave. Transactions ASCE, 118, pp. 575- 587, 1953.

[8] Lin, C., Ho, T. C., Chang, S. C., Hsieh, S. C., and Chang, K. A.,Vortex shedding induced by a solitary wave propagating over a submerged vertical plate. International Journal of Heat and Fluid Flow, 26, pp. 894-904, 2005. 